\title{
Surgical treatment of ascending aorta floating thrombus in a patient with recent SARS-CoV-2 infection
}

\author{
Igor Zivkovic ${ }^{1}$, Petar Milacic ${ }^{1}$, Vladimir Mihajlovic ${ }^{2}$, Stasa Krasic ${ }^{3}$, Jelena Lesanovic ${ }^{4}$, Miodrag Peric ${ }^{1}$, \\ Djordje Zdravkovic ${ }^{1}$ \\ ${ }^{1}$ Department of Cardiac Surgery, Dedinje Cardiovascular Institute, Belgrade, Serbia; ${ }^{2}$ Department of Radiology, Dedinje Cardiovascular Institute, \\ Belgrade, Serbia; ${ }^{3}$ Department of Cardiology, Mother and Child Health Institute of Serbia, Belgrade, Serbia; ${ }^{4}$ Department of Anesthesiology, \\ Dedinje Cardiovascular Institute, Belgrade, Serbia \\ Correspondence to: Igor Zivkovic, MD. Department of Cardiac Surgery, Heroja Milana Tepica 1, 11000 Belgrade, Serbia. \\ Email: igor88zivkovic@gmail.com.
}

\begin{abstract}
The 44-year-old female was admitted to the hospital due to the severe pain in the right arm. Doppler ultrasonography revealed occlusion of the right brachial and right common carotid artery. Subsequently, computed tomography (CT) scan confirmed the occlusion of the right brachial and common carotid artery, and revealed pedunculated floating ascending aortic mass. The floating thrombus in ascending aorta is a rare and potentially very dangerous pathological condition. Although aetiology is still unclear, ascending aorta atherosclerosis and coagulation disorder are probably the most common reasons. Hypercoagulable state, high level of antiphospholipid antibody and factor VIII are closely relating with vascular thrombosis. Since the outbreak of the coronavirus-2019 (COVID-19) pandemic, increasing evidence suggests that severe acute respiratory syndrome coronavirus 2 (SARS-CoV-2) might produce hypercoagulable state with subsequent thrombosis and thromboembolism. The infection elevated the level of factor VIII in the blood and, consequently, increased the risk of adverse thrombotic and embolic events. Additionally, endothelial inflammation and injury mediated by coronavirus represent an additional risk factor. According to literature, this is the first case of the floating ascending aorta thrombus in the patient with SARS-CoV-2 infection. The thrombus' fragile structure and high blood velocity through the ascending aorta significantly increases cerebral and peripheral embolization incidence, with potentially fatal outcome. Due to frequent adverse events, urgent surgical extirpation is the best therapy option. We presented successfully surgically treated giant floating thrombus in the ascending aorta and aortic arch in a patient with recent SARS-CoV-2 infection with acute right arm ischemia due to embolic complication.
\end{abstract}

Keywords: Ascending aorta; thrombus; aortic arch; embolization; SARS-CoV-2

Submitted Dec 16, 2020. Accepted for publication Jan 31, 2021.

doi: $10.21037 / \mathrm{cdt}-20-1010$

View this article at: http://dx.doi.org/10.21037/cdt-20-1010

\section{Introduction}

The ascending aorta floating thrombus is a rare pathological finding with potentially fatal embolic complication (1). Although atherosclerosis of ascending aorta and hypercoagulable disorders could be the main reason (2), the aetiology is unknown. Since the outbreak of the coronavirus-2019 (COVID-19) pandemic, increasing evidence suggests that severe acute respiratory syndrome coronavirus 2 (SARS-CoV-2) might produce hypercoagulable state with subsequent thrombosis and thromboembolism $(3,4)$. The relation between SARS$\mathrm{CoV}-2$ infection and ascending aorta thrombosis did not describe in the current literature. Unstable structure and high blood velocity in the ascending aorta could cause thrombus to dislodge into the supra-aortic or peripheral vessels. Urgent treatment is obligatory in this condition. Surgical evacuation and thrombolytic therapy are the main 

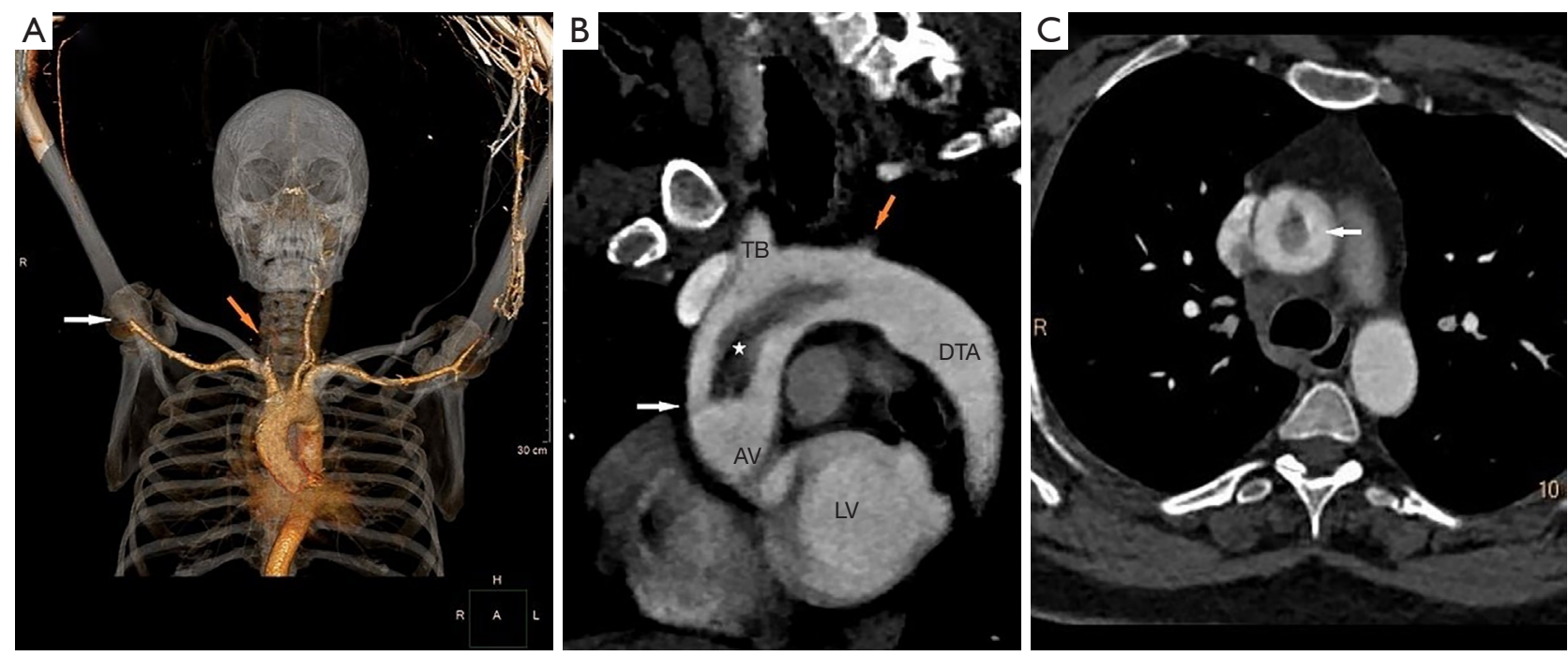

Figure 1 Computed tomography angiography (CTA) of the upper body and thorax. (A) Volume rendering 3D reconstruction-notice the occlusion of the right common carotid and right brachial artery. The white arrow indicates it occluded right brachial artery; orange arrow indicates it occluded right common carotid artery. (B) Pedunculated floating ascending aortic mass $(75 \mathrm{~mm} \times 12 \mathrm{~mm})$ arising from the sinotubular junction progressing into the aortic arch. White arrow, the place of the thrombus attachment to the wall of the aorta; asterisk, thrombus mass; orange arrow, left subclavian artery. (C) Axial view of ascending aorta-central filling defect surrounded with contrast. These findings are consistent with free-floating thrombus formation in ascending aorta. White arrow, thrombotic mass in the aorta. LV, left ventricle; AV, aortic valve; TB, truncus brachiocephalic; DTA, descending thoracic aorta.

therapeutic options (5).

We presented surgical extirpation of giant floating thrombus from the ascending aorta and aortic arch in a SARS-CoV-2 positive patient with acute right arm ischemia due to embolic complication.

We present the study in accordance with the CARE reporting checklist (available at http://dx.doi.org/10.21037/ cdt-20-1010).

\section{Case presentation}

The 44-year-old female was admitted to the hospital due to the severe pain in the right arm. Symptoms of pain, numbness and coldness of the right hand started two weeks before. Her medical history revealed previous myocardial infarction (two years before, without angiographic confirmation of the presence of coronary artery disease), and arterial hypertension (medically treated). She did not have a previous history of cerebral events.

According to our Institutional protocol based on the COVID-19 outbreak, SARS-CoV-2 specific neutralizing antibodies were measured at the admission. In the blood sample of the patient, IgM antibodies were detected by chromatography technique. However, the patient did not have any symptoms of acute viral infection in recent medical history.

Colour duplex scan of the right arm revealed total occlusion of the right brachial artery. In addition, total occlusion of the right common carotid artery was detected. Transthoracic echocardiography showed preserved contractile function of the left ventricle (ejection fraction $60 \%$, without any pathological findings on the valves. However, a vast floating mass was detected in the ascending aorta. It was attached to the aorta's sinotubular junction with protrusion into the aortic arch (Videos 1 and 2). Subsequently, computed tomography (CT) scan confirmed occlusion of the right brachial and common carotid artery (Figure 1A). Additionally, a sizeable peduncular tumour formation (measured as $75 \mathrm{~mm} \times 12 \mathrm{~mm}$ ) rose from the anterior wall of the aorta's sinotubular junction through the end of the aortic arch (Figure 1B,C) was described.

Due to the risk of embolization, an urgent surgical procedure was performed through a median sternotomy. The cardio-pulmonary bypass was instituted by right femoral artery and right atrial appendage cannulation, and the patient was cooled down to $20^{\circ} \mathrm{C}$. After stopping 

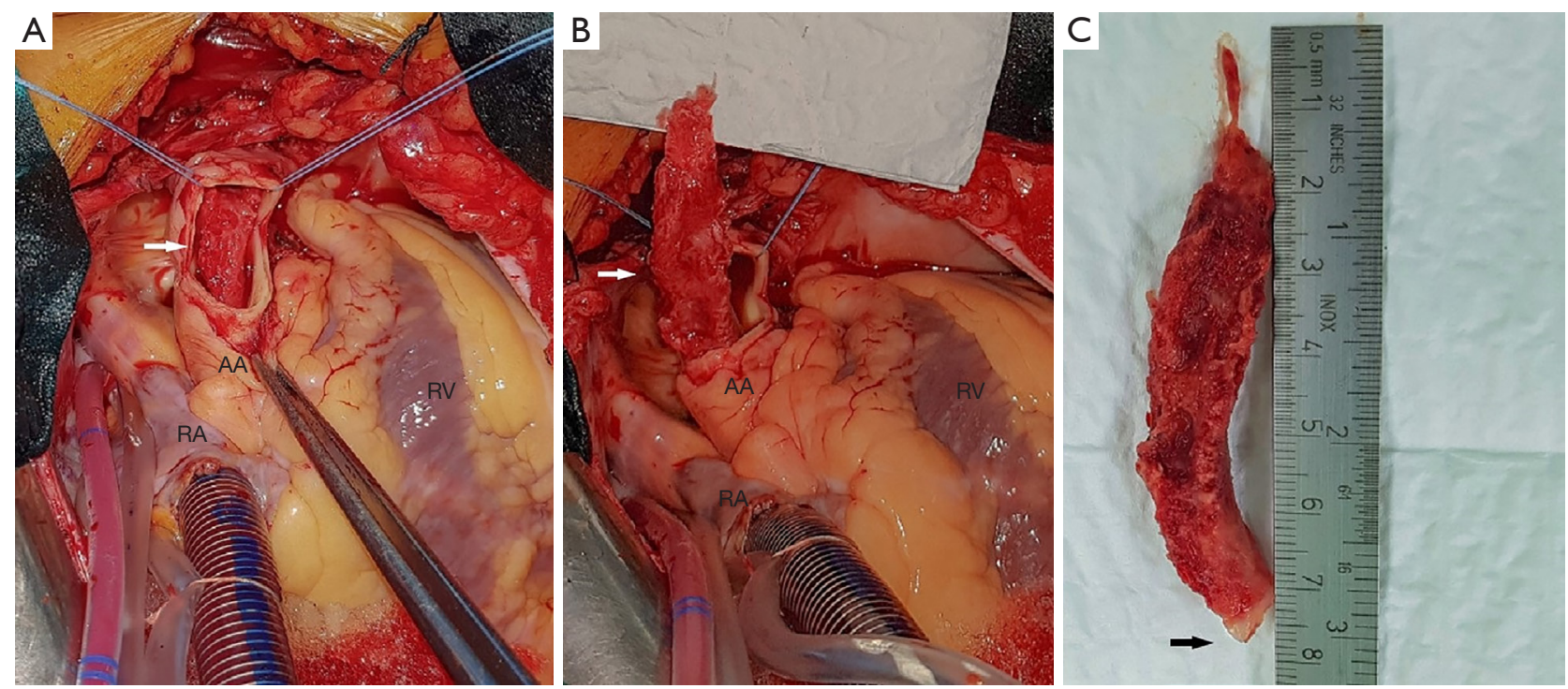

Figure 2 Surgical view of the intra-aortic, partial and wholly removed thrombus mass. (A) Intra-aortic presentation of the thrombus through the transverse aortotomy. (B) Distal part of thrombus extirpated from the aortic arch. (C) Completely extracted $80 \mathrm{~mm} \times 13 \mathrm{~mm}$ thrombus mass. White arrow, thrombus mass; black arrow, pedicle of thrombus. LV, left ventricle; RA, right atrium; AA, ascending aorta.

the circulation, transverse aortotomy at the level of sinotubular junction was performed, and intra-aortic tumour formation was found (Figure 2A). The distal part of the thrombus extended to the aortic arch (Figure $2 B$ ). The pedicle was transacted, and $80 \mathrm{~mm} \times 13 \mathrm{~mm}$ thrombus was removed entirely (Figure $2 C$ ). There was no significant atherosclerosis or any other sign of aortic disease in the area of thrombus attachment, but to prevent future recurrence, this part of ascending aorta was resected and replaced with Dacron tubular graft.

Oral anticoagulation treatment (warfarin) was initiated on the first postoperative day. The coagulation system screening (platelet count, bleeding time, prothrombin ratio, activated partial thromboplastin time, thrombin time, liver function tests) revealed an increased level of factor VIII (261 U/dL, normal range, 50-150 U/dL), while other procoagulants (coagulation factor I-XIII, lupus anticoagulant, anticardiolipin antibodies) and anticoagulants (Protein C, Protein S, anti-thrombin III) were in normal ranges. The pathohistological examination confirmed the extracted mass to be thrombus, excluding the presence of malignant cells. The postoperative period was uneventful, and the patient was discharged from the hospital on the sixth postoperative day.

All procedures performed in studies involving human participants were in accordance with the ethical standards of the institutional and/or national research committee(s) and with the Helsinki Declaration (as revised in 2013). Written informed consent was obtained from the patient for publication of this manuscript and any accompanying images.

\section{Discussion}

The floating thrombus in the ascending aorta is a rare pathological condition, which may cause severe embolic complications (1). The first case of young women with recurrent peripheral and cerebral embolic events was described in 1976 by Heiskell and Conn (6).

The aetiology of floating thrombus in ascending aorta is unclear, but atherosclerosis or debris on atherosclerotic plaques was described as the most frequent cause. Laperche et al. described 23 cases of floating thrombi of the aorta, and in 21 cases, pathological examination revealed small atherosclerotic plaques (7). Our patient had no significant atherosclerosis or any other sign of aortic disease in the area of thrombus attachment.

The first reported case of ascending aortic floating thrombus with no evidence of aortic atherosclerosis was a young woman who had systemic lupus erythematosus. Thrombus was caused by a hypercoagulable state induced by her illness (8). The antiphospholipid syndrome also induces 
the hypercoagulable state and increases the risk of thrombus formation (9). In the described case, coagulation screening detected a high level of antihemophilic factor A (factor VIII). However, other procoagulants and anticoagulants were in normal ranges; we ruled out antiphospholipid syndrome, systemic lupus, and malignancy as potential causes of hypercoagulable state in the presented patient. Many authors have recently demonstrated a strong link between COVID-19 infection and thromboembolism. In a study of 19 critically ill patients with COVID-19, elevated factor VIII levels were observed in $100 \%$ of participants (10). Factor VIII has an essential role in arterial thrombosis (3). The pathophysiology of thromboembolism in COVID-19 compared with non-COVID-19 state may be associated with viral-mediated endothelial inflammation, in addition to hypercoagulability connected with increased concentrations of coagulation factors $(10,11)$. Additionally, our patient had a transient hypercoagulable state, likely secondary to the recent SARS-CoV-2 infection. Woehl et al. presented caseserial of 4 patients with bilateral pulmonary ground glass opacities caused by SARS-CoV-2 and descending aortic thrombosis (11). To the best of our knowledge, that is the first case of a patient with recent asymptomatic SARS$\mathrm{CoV}-2$ infection and giant thrombus in ascending aorta.

Some authors suggest that aortitis, endothelial injury and dysfunction could be the main reasons for thrombosis $(2,4)$. Additionally, complement-mediated endothelial injury and endothelitis were described in SARS-CoV-2 positive patients due to direct viral acting on the angiotensinconverting enzyme two receptor (10-12).

Embolic events are the most common clinical presentation of the disease. Thrombus rising near to the sinuses of Valsalva may cause ischemic heart attack, whereas the mass, located in the ascending aorta leads to cerebral and peripheral embolic events, as well (2).

Thrombolytic treatment may lead to the complete thrombus resolution, but the risk of disruption and consequent embolic events is increased. Urgent surgical treatment might be a method of choice in treating this potentially deadly condition. Femoral arterial cannulation and hypothermic circulatory arrest are a safe strategy for thrombus in the ascending aorta and aortic arch (4).

In conclusion, floating ascending aortic thrombus is a hazardous condition with high potential of thromboembolic complications. The hypercoagulable state is a significant risk factor; consequently, the complete hematologic examination should be performed. The SARS-CoV-2 infection could be considered a significant risk factor, leading to coagulopathy and hypercoagulable state of blood with increased risk of vessels thrombosis and embolism. Urgent surgical extirpation is a gold standard since fragile thrombus presents the risk of embolic events.

\section{Acknowledgments}

Funding: None.

\section{Footnote}

Reporting Checklist: We present the study in accordance with the CARE reporting checklist. Available at http://dx.doi. org/10.21037/cdt-20-1010

Conflicts of Interest: All authors have completed the ICMJE uniform disclosure form (Available at http://dx.doi. org/10.21037/cdt-20-1010). The authors have no conflicts of interest to declare.

Ethical Statement: The authors are accountable for all aspects of the work in ensuring that questions related to the accuracy or integrity of any part of the work are appropriately investigated and resolved. All procedures performed in studies involving human participants were in accordance with the ethical standards of the institutional and/or national research committee(s) and with the Helsinki Declaration (as revised in 2013). Written informed consent was obtained from the patient for publication of this manuscript and any accompanying images.

Open Access Statement: This is an Open Access article distributed in accordance with the Creative Commons Attribution-NonCommercial-NoDerivs 4.0 International License (CC BY-NC-ND 4.0), which permits the noncommercial replication and distribution of the article with the strict proviso that no changes or edits are made and the original work is properly cited (including links to both the formal publication through the relevant DOI and the license). See: https://creativecommons.org/licenses/by-nc-nd/4.0/.

\section{References}

1. Jaworski L, Fijalkowski M, Rogowski J. Giant thrombus in ascending aorta and aortic arch. J Thorac Cardiovasc Surg 2013;145:1668-9.

2. Calderon P, Heredero A, Pastor A, et al. Successful removal of a floating thrombus in ascending aorta. Ann 
Thorac Surg 2011;91:e67-9.

3. Helms J, Tacquard C, Severac F, et al. High risk of thrombosis in patients with severe SARS-CoV-2 infection: a multicenter prospective cohort study. Intensive Care Med 2020;46:1089-98.

4. Shi W, Lv J, Lin L. Coagulopathy in COVID-19: Focus on vascular thrombotic events. J Mol Cell Cardiol 2020;146:32-40.

5. Choi JB, Choi SH, Kim NH, et al. Floating thrombus in the proximal aortic arch. Tex Heart Inst J 2004;31:432-4.

6. Heiskell CA, Conn J Jr. Aortoarterial emboli. Am J Surg 1976;132:4-7.

7. Laperche T, Laurian C, Roudaut R, et al. Mobile thromboses of the aortic arch without aortic debris. A transesophageal echocardiographic finding associated with unexplained arterial embolism. The Filiale Echocardiographie de la Société Française de Cardiologie.
Circulation 1997;96:288-94.

8. Kalangos A, Baldovinos A, Vuille C, et al. Floating thrombus in the ascending aorta: a rare cause of peripheral emboli. J Vasc Surg 1997;26:150-4.

9. Ryu YG, Chung CH, Choo SJ, et al. A case of antiphospholipid syndrome presenting with a floating thrombus in the ascending aorta. J Thorac Cardiovasc Surg 2009;137:500-2.

10. Piazza G, Morrow DA. Diagnosis, Management, and Pathophysiology of Arterial and Venous Thrombosis in COVID-19. JAMA 2020;324:2548-9.

11. Woehl B, Lawson B, Jambert L, et al. 4 Cases of Aortic Thrombosis in Patients With COVID-19. JACC Case Rep 2020;2:1397-401.

12. Hostetter L, Aneesah Jaumally B, et al. COVID-19associated hypercoagulable state: a case of acute aortic thrombosis. Chest 2020;158:A870.

Cite this article as: Zivkovic I, Milacic P, Mihajlovic V, Krasic S, Lesanovic J, Peric M, Zdravkovic D. Surgical treatment of ascending aorta floating thrombus in a patient with recent SARS-CoV-2 infection. Cardiovasc Diagn Ther 2021;11(2):467-471. doi: 10.21037/cdt-20-1010 